\title{
The Effect Analysis of Wind Load on Vehicle-Bridge Coupled Dynamic Behavior \\ LI Yan ${ }^{1, a}$ and SUN Hang ${ }^{1, b}$
}

\author{
${ }^{1}$ School of Transportation Science and Engineering, Harbin Institute of Technology, Harbin, China \\ aliyan_2007@126.com, bridge_hit@126.com
}

\begin{abstract}
Keywords: Vehicle-Bridge Coupled Vibration; Mode Integration Theory; Wind Load; Buffeting.
\end{abstract}
\begin{abstract}
A computer simulation method of solving vehicle-bridge system vibration response considering wind load is presented based on mode integration theory. Vehicle model and fluctuating wind load model are established, corresponding vehicle stiffness and damping matrix is educed in the method. A practical cable-stayed bridge dynamic analysis is completed successfully by programming with fore mentioned method. Wind load have a great influence on bridge vibration acceleration under low wind velocity. Vehicle vertical dynamic response is influenced evidently by wind induced bridge vibration, its lateral response is controlled by wind load and bridge response. The effect on system dynamic response of wind load is remarkable. For other type large span bridges the method also can be referred and have important application value in interrelated research. The accuracy of calculation is high enough for practical purposes.
\end{abstract}

\section{Introduction}

Long span cable-stayed bridges mostly were used crossed large river and channel, where hurricane usually happened. Hurricane-induced strong winds have been greatly threatening the safety of vehicles on the bridge. Currently, decisions on driving speed limits and closing of transportation on bridges and highways in windy environments are mostly based on intuition or subjective experience [1]. Durative strong wind load also affect the structural durability and safety of bridge. A rational prediction of the performance of vehicle-bridge system under strong winds is of utmost importance to ensure transportation efficiency and safety [2].

Recently the study on vehicle-bridge vibration under strong wind is rarely carried out [2]. Hence the research for vehicle-bridge system dynamic response under strong wind is indispensable for guaranteeing bridge operation security and transportation safety.

The present study put forward a vehicle-bridge dynamic response analysis method considering dynamic wind load based on the mode integration theory. Dynamic equilibrium equation derivation of vehicle model and wind load model are given in the paper. The project example analysis shows that the method is suitable for the interrelated analysis of similar engineering and project appliance.

\section{Simulation of stochastic wind field}

The vehicle-bridge coupling vibration system dynamic response is analyzed in time domain for its obvious time-varying characteristic. Simulation of stochastic wind field is first step to compute vehicle-bridge vibration under wind circumstance. In the study spectral representation method is used to simulate of stochastic wind field. Supposed wind speed and wind spectrum along bridge length direction are invariable, bridge deck heights are in is uniform, the distance of two closed wind speed simulate point is same. Impulsive wind load of the $\mathrm{j}$ point can be generated with:

$$
f_{j}(t)=2 \sqrt{\Delta \omega} \sum_{m=1}^{j} \sum_{l=1}^{N}\left|H_{j m} \omega_{m l}\right| \cos \left(\omega_{m l} t-\theta_{j m}\left(\omega_{m l}\right)+\phi_{m l}\right), \quad(j=1,2, \cdots, m)
$$

where $\Delta \omega=\left(\omega_{\max }-\omega_{\min }\right) / N, \omega_{l}=\omega_{\min }+(l-1 / 2) \Delta \omega, N$ is frequency interval, $\omega_{\max }, \omega_{\min }$ are maximal and minimal frequency limited value, $\phi_{m l}$ is a random variable uniformly distributed between 0 and $2 \pi ; S$ is cross-spectral density matrix of wind spectrum, $H$ is Cholesky decomposition matrix of $S$. $H_{j m}$ is the lower triangular matrix of $(j, m)$ subdeterminant of $H$ :

$$
\theta_{j m}\left(\omega_{m l}\right)=\tan ^{-1}\left(\frac{\operatorname{Im} H_{j m}\left(\omega_{m l}\right)}{\operatorname{Re} H_{j m}\left(\omega_{m l}\right)}\right)
$$


Simu spectrum is used in simulating wind load in lateral and along bridge directions, LumleyPanofsky spectrum is used in vertical, defined equations can be found in [3]. The author completed simulation stochastic wind field of the long span cable-stayed bridge with self-compiling computing program.

\section{Dynamic analysis of vehicle-bridge system under strong wind}

\subsection{Vehicle model}

In the present study, a vehicle is modeled as a combination of several rigid bodies connected by several axle mass blocks, springs, and damping devices (Fig.1). The bridge deck and the tires of the vehicles are assumed to be point-contact without separation. Vehicle body owns four DOF: vertical displacement, lateral displacement, pitching displacement and rolling displacement; each wheel owns vertical and lateral displacement; the whole vehicle model includes 12 DOFs.
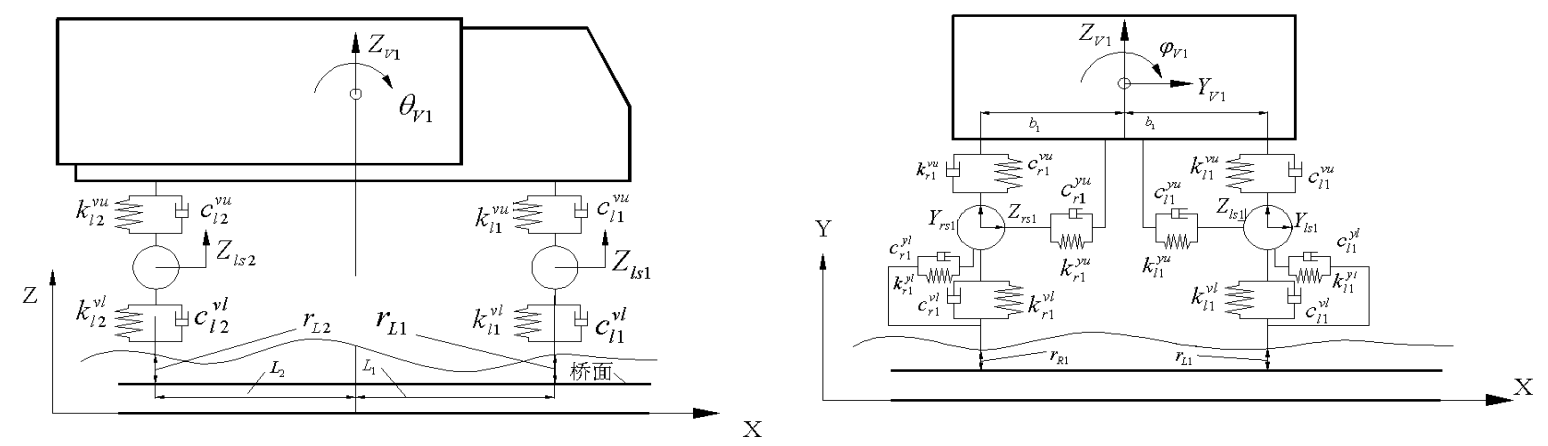

Fig .1 Vehicle model

The vehicle stiffness matrix $K_{v}$ is detruded by stiffness integration method. Both the stiffness and damping matrix are symmetrical. $K_{v}$ and $C_{v}$ have same form, $C_{v}$ can be gotten by displacing $k$ with $c$. Vehicle stiffness matrix are listed in reference [6]

\subsection{Bridge model}

The long span and complexion of factual bridge structure make compute vehicle-bridge system vibration difficulty and calculate cost. The study shows that dynamic response of complex bridge structure is controlled by its several low order mode. The structure dynamic analysis accuracy can arrive engineering requirements by considering several low order modes. In the present study mode integration theory is applied in vehicle-bridge vibration analysis. At first cable-stayed bridge FEM is built, construction mode and frequency can be attained by modal analysis. Motions in three directions including vertical, lateral, and torsion of the bridge are expressed with the mode superposition technique as:

where $\phi_{h}^{n}(x), \phi_{\theta}^{n}(x), \phi_{v}^{n}(x)$ respectively are the components in the three directions (lateral, vertical and torsion) for the $n$th mode shape; $N_{b}$ is the total number of modes for the bridge under consideration; $q_{n}$ is the generalized coordinates of the bridge. All the mode shapes are standardized by generalized mass equaling 1 . The displacement of bridge can be showed as:

$$
Y_{b}(x)=\sum_{n=1}^{N_{b}} q_{n} \phi_{h}^{n}(x), \theta_{b}(x)=\sum_{n=1}^{N_{b}} q_{n} \phi_{\theta}^{n}(x), Z_{b}(x)=\sum_{n=1}^{N_{b}} q_{n} \phi_{v}^{n}(x)
$$

\subsection{Vehicle-bridge system motion equation under strong wind load}

The wind effects on vehicle-bridge system consist of lift force, drag force and rolling moment, each component includes static force, aeroelastic self-excited forces induced by interaction between wind and bridge or vehicle, and turbulent buffeting forces. It is usually assumed that the analysis begins with the static equilibrium position of the bridge under self-weight and static wind force action. Only aeroelastic self-excited forces and turbulent buffeting forces on the bridge, static wind and turbulent buffeting forces on the vehicle are considered in the dynamic analysis of the bridge. 
The motion equations of vehicle-bridge system under wind load are:

$$
\left[\begin{array}{cc}
M_{v v} & 0 \\
0 & M_{b b}
\end{array}\right]\left\{\begin{array}{l}
\ddot{X}_{X^{v}} \\
\ddot{X}_{b}
\end{array}\right\}+\left[\begin{array}{cc}
C_{v v} & C_{v b} \\
C_{b v} & C_{b b}
\end{array}\right]\left\{\begin{array}{c}
\dot{X}_{v} \\
\dot{X}_{b}
\end{array}\right\}+\left[\begin{array}{cc}
K_{v v} & K_{v b} \\
K_{b v} & K_{b b}
\end{array}\right]\left\{\begin{array}{l}
X_{v} \\
X_{b}
\end{array}\right\}=\left\{\begin{array}{l}
F_{v b} \\
F_{b v}
\end{array}\right\}+\left\{\begin{array}{l}
F_{v}^{s t}+F_{v}^{b f} \\
\tilde{F}_{b}^{b f}+\tilde{F}_{b}^{s e}
\end{array}\right\}
$$

where $M, K, C$ respectively represent for mass, stiffness and damping matrix; $X, \dot{X}, \ddot{X}$ represent for displacement, velocity and acceleration respectively; superscripts of " $v$ " refer to vehicle, " $b$ " refer to bridge, " $v b$ " and " $b v$ " refer to the vehicles-bridge coupled terms; $F_{v}^{s t}, F_{v}^{b f}$ represent for static and turbulent buffeting forces on the vehicles respectively; $\tilde{F}_{b}^{b f}, \tilde{F}_{b}^{s e}$ represent for modal turbulent buffeting forces and self-excited forces on the bridge respectively. The matrix form of vehicles-bridge interaction can be found in [4]. Newmark- $\beta$ method is used to solve the system motion equations. The wind load on the vehicles-bridge system is discussed in the following section.

\subsection{Wind load on the vehicles-bridge system}

\subsubsection{Wind load on bridge}

(1) Buffeting forces

The buffeting forces on the ith point of the bridge is expressed as:

$$
F_{b i}^{b f}=\left[f_{D i}^{b f}, f_{M i}^{b f}, f_{L i}^{b f}\right]^{T}
$$

where: $\begin{aligned} f_{D i}^{b f} & =0.5 \rho \bar{U}^{2} B L_{i}\left[2 C_{D i} u_{i}(t)+C_{D i}^{\prime} w_{i}(t)\right], f_{M i}^{b f}=0.5 \rho \bar{U} B^{2} L_{i}\left[2 C_{M i} u_{i}(t)+C_{M i}^{\prime} w_{i}(t)\right] \\ f_{L i}^{b f} & =0.5 \rho \bar{U} B L_{i}\left[2 C_{L i} u_{i}(t)+\left(C_{L i}^{\prime}+C_{D i}\right) w_{i}(t)\right]\end{aligned}$

where $u_{i}(t), w_{i}(t)$ represent horizontal and vertical component of turbulent wind respectively; $f_{D i}^{b f}, f_{M i}^{b f}, f_{L i}^{b f}$ are buffeting drag force, torsion moment and lift force on the $i$ th point of the bridge; $\rho$ is air dense; $\bar{U}$ refer to mean wind velocity; $B_{i}, L_{i}$ represent section width and height of the $i$ th girder section; $C_{L}, C_{M}, C_{D}$ and $C_{L}^{\prime}, C_{M}^{\prime}, C_{D}^{\prime}$ refer to aerodynamic coefficient and corresponding Derivatives.

(2) Self-excited forces on the bridge

The self-excited forces can be expressed as product of displacement and impulse response function of the bridge in the time domain, the impulse response function are educed by the function of flutter derivative from wind tunnel experiment[5]. The self-excited force on the $i$ th point of bridge is expressed as:

$$
\begin{aligned}
& f_{L i}^{\text {se }}(t)=\frac{1}{2} \rho \bar{U}_{i}^{2} B_{i} L_{i}\left\{A_{D a 1} a_{i}(t)+A_{D a 2}\left(\frac{B_{i}}{\bar{U}_{i}}\right) \dot{a}_{i}(t)+A_{D a 3}\left(\frac{B_{i}}{\bar{U}_{i}}\right)^{2} \ddot{a}_{i}(t)+\sum_{k=1}^{m} A_{D a k+3} \int_{-\infty}^{t} \dot{a}_{i}(\tau) \exp \left[-\frac{d_{D a k}}{B_{i}}(t-\tau)\right] d \tau\right\} \\
& +\frac{1}{2} \rho \bar{U}_{i}^{2} L_{i}\left\{A_{D h 1} h_{i}(t)+A_{D h 2}\left(\frac{B_{i}}{\bar{U}_{i}}\right) \dot{h}_{i}(t)+A_{D h 3}\left(\frac{B_{i}}{\bar{U}_{i}}\right)^{2} \ddot{h}_{i}(t)+\sum_{k=1}^{m} A_{D h k+3} \int_{-\infty}^{t} \dot{h}_{i}(\tau) \exp \left[-\frac{d_{D h k}}{B_{i}}(t-\tau)\right] d \tau\right\} \\
& +\frac{1}{2} \rho \bar{U}_{i}^{2} L_{i}\left\{A_{D p 1} p_{i}(t)+A_{D p 2}\left(\frac{B_{i}}{\bar{U}_{i}}\right) \dot{p}_{i}(t)+A_{D p 3}\left(\frac{B_{i}}{\bar{U}_{i}}\right)^{2} \ddot{p}_{i}(t)+\sum_{k=1}^{m} A_{D p k+3} \int_{-\infty}^{t} \dot{p}_{i}(\tau) \exp \left[-\frac{d_{D p k k}}{B_{i}}(t-\tau)\right] d \tau\right\}
\end{aligned}
$$

Where $p_{i}(t), a_{i}(t), h_{i}(t)$ refer to lateral, torsional and vertical displacement of the $i$ th point of bridge respectively; $A_{D a j}, A_{D p j}, A_{D h j}(j=1, \cdots, 3+m)$ and $d_{D a k}, d_{D a k}, d_{D a k}(k=1,2, \cdots, m)$ are nondimensional coefficient which are irrespective with frequency and can be obtained by least squares method based on actual measured flutter derivative under different reduced frequency. $m$ usually equate 2 . Self-excited lift force and torsional moment expression can be gotten by the same method. 


\subsubsection{Wind load on the vehicle}

Showed in fig.2, mean wind speed on bridge is $U_{m}$, the driving speed of vehicle is $U_{v}$, the relative wind speed can be expressed as:

$U_{R}=\sqrt{[\bar{U}+u(x, t)]^{2}+U_{V}^{2}}, \varphi=\operatorname{arctg}\left[\frac{\bar{U}+u(x, t)}{U_{V}}\right]$

Where $u(x, t)$ represents turbulent wind speed component on the vehicle, which can be simulated by the same method with bridge. The static wind and buffering force on the vehicles are expressed as:

$$
\begin{aligned}
& F_{x}=0.5 \rho U_{R} C_{D}(\varphi) A_{f} ; F_{y}=0.5 \rho U_{R}^{2} C_{S}(\varphi) A_{f} ; F_{z}=0.5 \rho U_{R}^{2} C_{L}(\varphi) A_{f} ; \\
& M_{x}=0.5 \rho U_{R}^{2} C_{R}(\varphi) A_{f} h_{v} ; M_{y}=0.5 \rho U_{R}^{2} C_{P}(\varphi) A_{f} h_{v} ; M_{z}=0.5 \rho U_{R}^{2} C_{Y}(\varphi) A_{f} h_{v} ;
\end{aligned}
$$

where $A_{f}$ is the frontal area of the vehicle; $h_{v}$ is the distance from the gravity center of the vehicle to the road surface; $C_{D}, C_{S}, C_{L}, C_{R}, C_{P}, C_{Y}$ are the coefficients of drag force, side force, lift force, rolling moment, pitching moment and yawing moment for the vehicle.

\section{Numerical example}

The cable-stayed bridge of the 2nd Changjiang river bridge of Nanjing has a main span $628 \mathrm{~m}$ and total length $1238 \mathrm{~m}$, its tower height is $195.41 \mathrm{~m}$. The FEM of the bridge is shown in fig.3. The dynamic response of vehicles-bridge system under strong wind for the bridge is studied by self-compiling program in Matlab. Dynamic characteristic calculation can be found in [6].

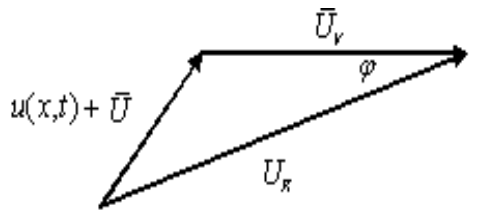

Fig .2 Relative wind velocity on the vehicle

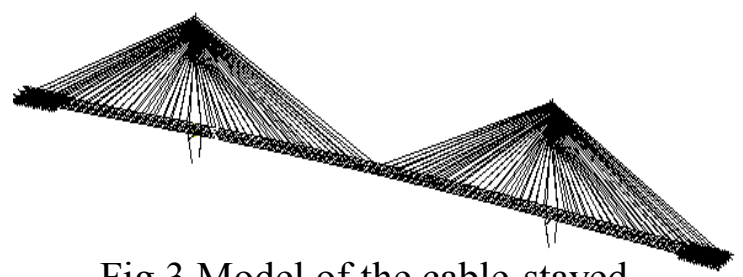

Fig. 3 Model of the cable-stayed bridge

In the dynamic analysis 20 low order modes is considered to meet computing accuracy requirement. The damping ratio of the bridge is defined as $0.5 \%$ on the dynamic field test. The total weight $300 \mathrm{kN}$ vehicle model is used in the present study. The model parameter and aerodynamic parameter are listed in the reference [7]. Take the road surface roughness as "good" level, roughness coefficient is $0.62 \times 10^{-6} \mathrm{~m}^{3} /$ cycle. The design wind speed is $40 \mathrm{~m} / \mathrm{s}$ on the girder height, the earth's surface roughness type is I level, the coherence function of wind spectrum is defined with Davenport
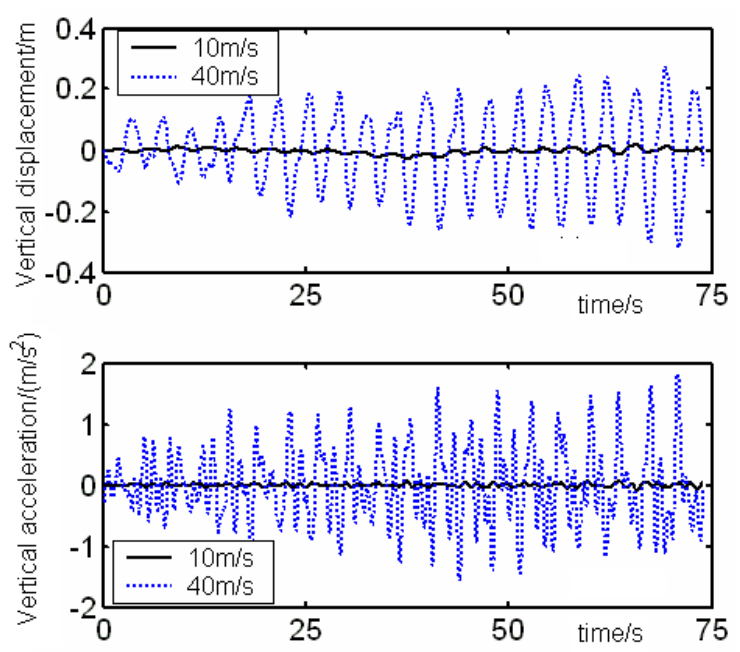

Fig.4 Vertical dynamic response of cable-stayed bridge in mid span
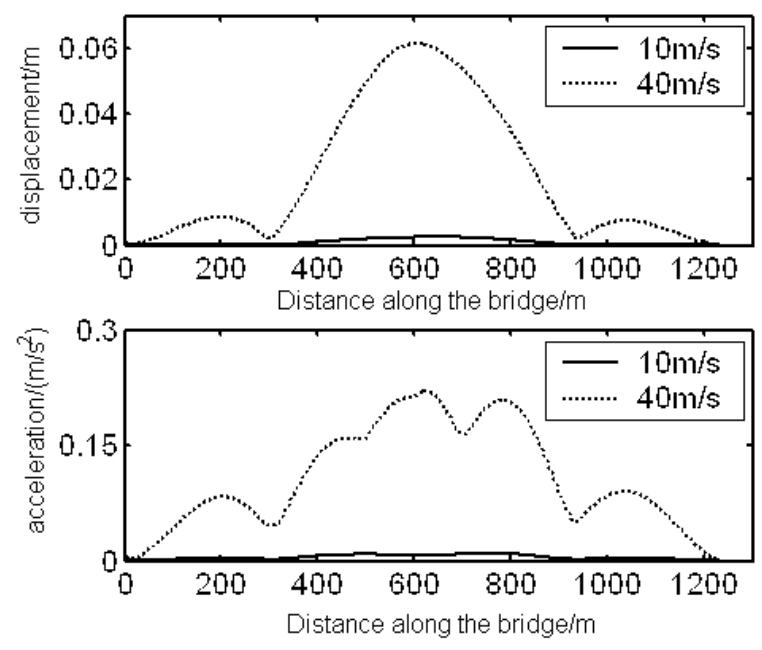

Fig. 5 Vertical peak value response of the bridge along span 
form [1], dimensionless deamplification factor is 7 , the distance of simulation points is $15.475 \mathrm{~m}$, aerodynamic parameters are attained by tunnel test [8]. Vehicle speed is $60 \mathrm{~km} / \mathrm{h}$, integral time length is $0.025 \mathrm{~s}$. In the study vehicle-bridge system dynamic response of the cable-stayed bridge is computed under $\bar{U}=10 \mathrm{~m} / \mathrm{s}$ and $\bar{U}=40 \mathrm{~m} / \mathrm{s}$ respectively. The analysis results are shown in Fig.4 to Fig.9.

Lateral response of the bridge is showed in Fig. 6 and Fig.7. It is suggested that wind load is main factor on lateral response of the bridge under strong wind, the point is validated as Fig. 7.
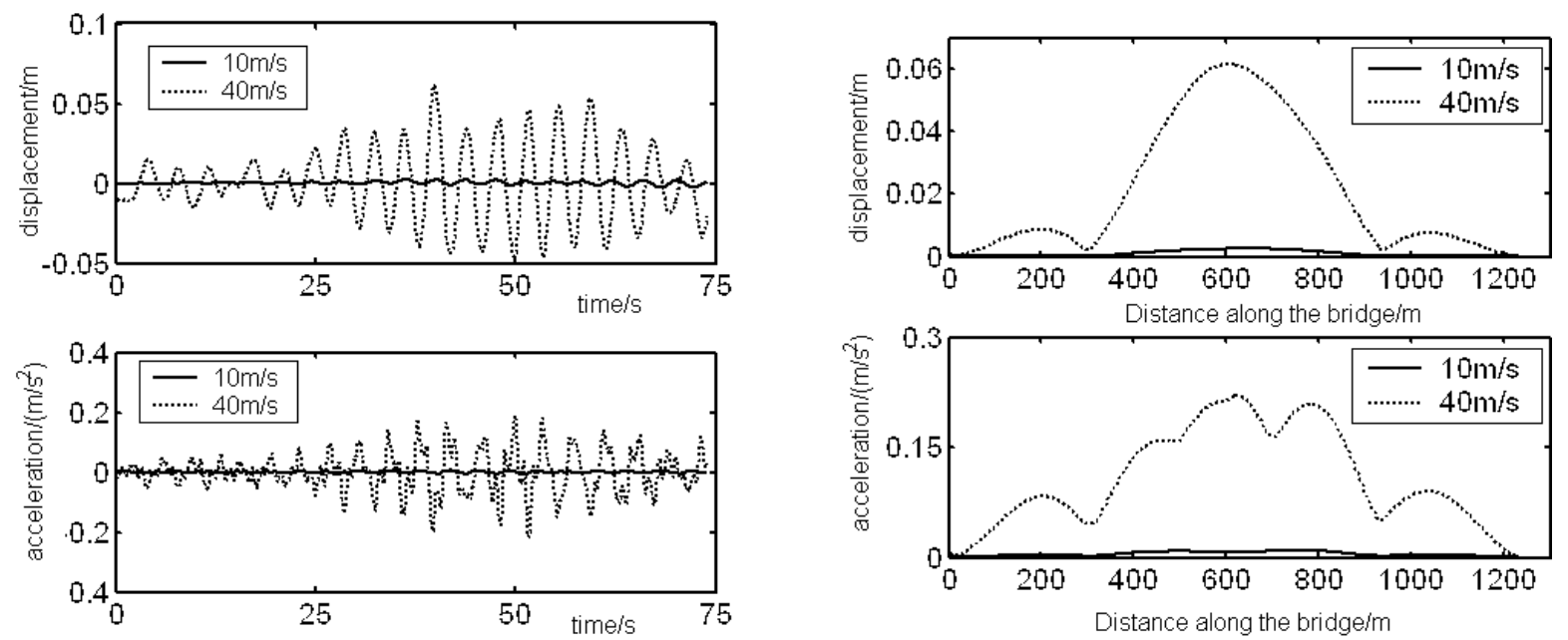

Fig. 6 Lateral response time history of in mid span

Fig. 7 Lateral peak value response along span
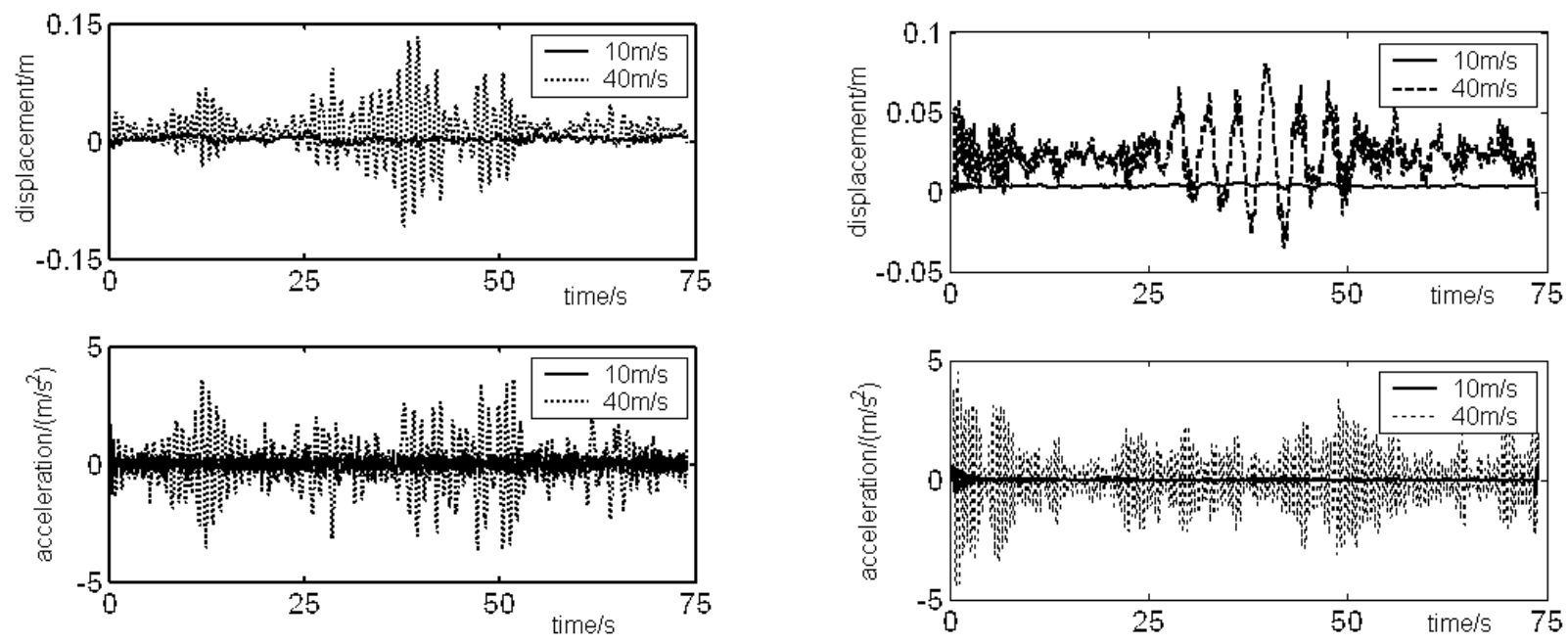

Fig. 8 Vertical response histories of vehicle body

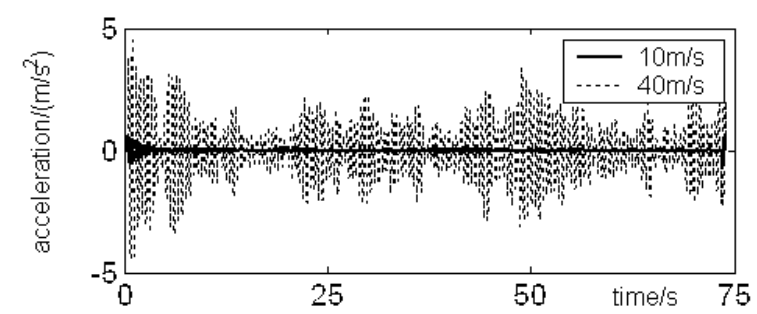

Fig.9 Lateral response histories of vehicle body

The displacement and acceleration time history in mid span of the bridge under two cases is shown in Fig.4. The vertical displacement and acceleration in mid span is controlled with wind load (wind speed $40 \mathrm{~m} / \mathrm{s})$; under relatively low wind speed $(10 \mathrm{~m} / \mathrm{s})$, the peak vertical displacement appears when vehicles driving through the mid of bridge, the acceleration has the same trend with displacement response, that indicates response is mainly affected by vehicle; The entire bridge displacement and acceleration peak appears peak when the vehicle is driven through the near mid of bridge, which indicates that the influence response comparison is showed in Fig.5, that indicates that wind load is the controlling factor for the vertical response of the bridge replacing vehicles under strong wind.

The vehicle response is showed in Fig.8 and Fig.9. Vertical displacement and acceleration of vehicle of bridge vibration on vertical dynamic response of vehicle is remarkable. As showed in Fig.9, the influence of instantaneous wind on vehicle response is greater when vehicle driving in the bridge. Vehicle displacement appears peak when it pass the middle of the bridge and then attenuate gently, the peak is caused by interaction of lateral vibration of the bridge in mid span and vehicle. It is observed that lateral dynamic response of vehicle is controlled with wind load by comparing results of relatively low and high wind speed cases. 


\section{Conclusion}

(1) The vehicle-bridge system dynamic response analysis model considering fluctuating wind is brought forward based on the mode integration theory. This method can be used for the relevant dynamic analysis of other types of long-span bridges.

(2) Analysis results showed that: the vertical displacement response of the bridge under strong winds was affected significantly by wind load, lateral displacement response was controlled by the wind load; under low wind speed $(10 \mathrm{~m} / \mathrm{s})$ the acceleration of the bridge was greater impacted by wind load, the effluence of bridge vibration on vertical displacement and acceleration response of the vehicle was obvious caused by wind load. The study showed that the safety of vehicle and bridge would be threaten under the strong wind conditions. Carrying out dynamic response study of vehicles-bridge under wind circumstance is very necessary.

(3) The study provides useful referring information and laid the initial foundation for related researches, including the further fatigue damage analysis of cable-stayed bridge under traffic and wind loads, vehicles safety driving on the bridge under disastrous wind load, and etc.

\section{Acknowledgements}

This research is financially supported by National Science Foundation of China (Grant Nos. 51108132) and Fundamental Research Funds for the Central Universities National Science Foundation of China (HIT.NSRIF.2012059).

\section{References}

[1] Z.X. Li, T.H.T. Chan and J.M. Ko: Fatigue Damage Model for Bridge under Traffic Loading: Application Made to Tsing Ma Bridge. Theoretical and Applied Fracture Mechanics, 2001, 35(3): 81-91.

[2] C.S. Cai, S.R. Chen. Framework of Vehicle-bridge-wind Dynamic Analysis. Journal of Wind Engineering and Industrial Aerodynamics, 2004, 92(2): 579-607.

[3] Li Yong-le, Zhou Shu-hua, Qiang Shi-zhong. Simulation of Three-dimensional Fluctuating Wind Field for Large Span Cable-stayed Bridge. China Civil Engineering Journal, 2003,36(10):60-65.

[4] W. H. GUO, Y. L. XU. Fully Computerized Approach to Study Cable-stayed Bridge-Vehicle Interaction. Journal of Sound and Vibration, 2001, 248(4): 745-761.

[5] Chen. X. Z, Matsumoto M, Kareem A. Time Domain Flutter and Buffeting Response Analysis of Bridges. Engineering Mechanics, ASCE, 2000, 126(1): 7-16.

[6] Li Yan. Wind-vehicle-bridge Dynamic Response and Fatigue Reliability of Cables of Long Span Cable-stayed Bridges. Ph.D dissertation, Harbin, Harbin Institute of Technology, 2008

[7] Sheng Guo-gang, Li Chuan-xi, Zhao Bing. Dynamic Analysis of A Simply-supported Beam Subjected to Moving Vehicles. Engineering Mechanics, 2006, 22(12):154-158.

[8] Wind Tunnel Laboratory of Tongji University. Wind Tunnel Experiment Report of the 2nd Nanjing Changjiang River Bridge. 1998 